MATEC Web of Conferences 11,03009 (2014)

DOI: $10.1051 /$ matecconf / 20141103009

(C) Owned by the authors, published by EDP Sciences, 2014

\title{
Influence of Grain Size Coarse Soil on Shear Strength
}

\author{
Ghembaza Moulay smaîne ${ }^{1}$, Birama Gueye ${ }^{1}$, Benamar Zoubir $^{1}$ \\ ${ }^{1}$ Faculté de Technologie, Laboratoire de Génie Civil et Environnement, Sidi Bel-Abbès, Algérie
}

\begin{abstract}
The geo- mechanical behavior of coarse soils is difficult to grasp by geotechnical methods, mainly due to the presence of large elements that disrupt or prevent the tests. Our work presents a study of the mechanical behavior of two coarse soils of different types namely Tuff MEKKEDRA and crushed gravel SIDI ALI- Benyoub west of Algeria by observing the effect of the size of diameters of the grains on the shear strength. Two aspects are considered. The first relates to a state of the art parameters which have a direct influence on the shear strength of coarse material. The second part concerns a presentation and analysis of the results of direct shear tests of materials prepared for Optimum Proctor. It is noted that the grain size affects in a significant way the properties of the shear strength. The bigger the diameter, the greater the angle of friction is important. However, the cohesion decreases with increasing grain size .
\end{abstract}

\section{Introduction}

Les matériaux naturels grossiers ont été utilisés dans les années 50 et 60 pour la construction de barrage, ensuite leur utilisation a été pratiquement abandonnée. Les sols grossiers sont souvent rencontrés dans la nature, en particulier dans les zones montagneuses. Ils sont constitués d'un mélange d'éléments de natures différentes et de tailles variables, du gros éléments appelés inclusions (cailloux, graviers...) jusqu'au fin (sable, limon, argile).

La réalisation d'un tel ouvrage est conditionnée par la compréhension du comportement des sols de fondation ou des sols constitutifs de l'ouvrage induit par leur hétérogénéité géologique. Plusieurs chercheurs ont étudié l'influence de différents paramètres liés aux dispositifs expérimentaux $[1,2,3,4,5,6,7,8,9,10,11]$ et à la nature des sols grossiers [12, 13, 14, 15, 16, 17]; [9] et [10] sur les caractéristiques mécaniques au laboratoire et in situ en vraie grandeur. La connaissance des valeurs de l'angle de frottement interne $\phi$ et de la cohésion c est nécessaire, voire indispensable pour le dimensionnement d'ouvrages de génie civil comme les soutènements, les fondations, les tunnels, les remblais. Ainsi, [17] mentionnent dans le cadre des ouvrages de protection contre les risques naturels, le dimensionnement est adopté par les valeurs moyennes des caractérisations mécaniques physiques des sols (cohésion nulle, angle de frottement $\phi^{\prime}=30^{\circ}$, contrainte admissible en portance sous charge verticale centrée $\sigma_{\mathrm{adm}}=0.2 \mathrm{MPa}$ et valeur unique du poids volumique des terres. Dans les formations de pente, et plus généralement dans les sols montagneux, ces données géotechniques sont peu nombreuses et les méthodes de reconnaissance classiques permettant d'y accéder (essais au laboratoire ou in situ) sont peu adaptées aux granulométries observées. L'amélioration de la connaissance des caractéristiques géomécaniques des sols grossiers constitue donc un axe de recherche important.

[5] a réalisé des essais de cisaillement direct dans des boites carrées de $1,2 \mathrm{~m}$ de côté et rondes de $100 \mathrm{~mm}$ de diamètre, sur un grès très friable de $160 \mathrm{~mm}$ de diamètre avec un ciment siliceux où ils étudier le rôle de la taille de l'appareil à celui du diamètre de la plus grande particule $\left(\mathrm{d}_{\max }\right)$. Ils ont mis en évidence la forte influence de la taille de l'appareil sur les résultats obtenus. On constate une décroissance de l'angle de frottement quand $\left(\mathrm{L} / \mathrm{d}_{\max }\right)$ augmente (Figure 1). Autrement dit lorsque le diamètre de la particule diminue.

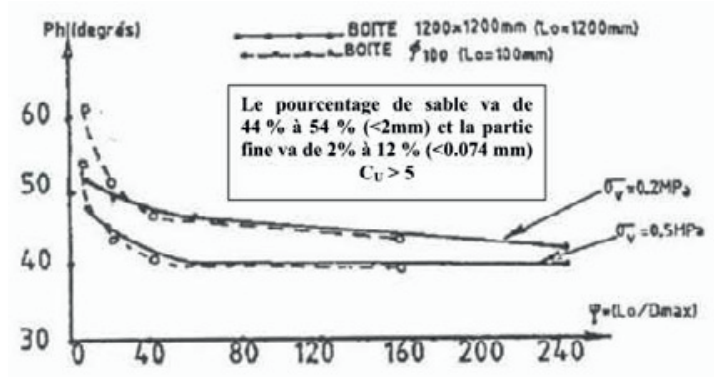

Fig. 1. Angle de frottement interne en fonction du facteur d'échelle (Fry et Flavigny, 1989)

[9] a étudié l'influence de la résistance au cisaillement en fonction de la taille de la boîte pour des sols écrêtés dont le $\mathrm{d}_{\max }$ est $25 \mathrm{~mm}$, à l'aide de trois boîtes de cisaillement à des longueurs des côtés qui valent respectivement 100 $\mathrm{mm}, 250 \mathrm{~mm}$ et $500 \mathrm{~mm}$. Il observe que les deux plus

This is an Open Access article distributed under the terms of the Creative Commons Attribution License 2.0, which permits unrestricted use, distribution, and reproduction in any medium, provided the original work is properly cited. 
grandes éprouvettes (celles dont la longueur des côtés sont 25 et 50 fois plus grandes que $d_{\max }$ ont des caractéristiques de rupture à peu près identiques alors que les paramètres de rupture évalués en testant la petite éprouvette de $100 \mathrm{~mm}$ de côté sont plus élevés (Figure 2).
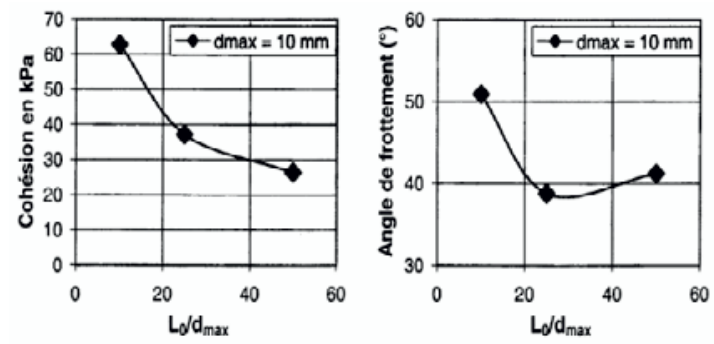

Fig. 2. Variation de l'angle de frottement interneet de la cohésion en fonction du rapport de taille $\left(\mathrm{L} / \mathrm{d}_{\max }\right)$ (Valle, 2001)

Par ailleurs, [1] a réalisé des essais triaxiaux avec un diamètre de l'éprouvette de $100 \mathrm{~mm}$ sur un granulat calcaire destiné à une industrie cimentaire de Bergame (Figure 3). Il montre une réduction de l'angle de l'angle de frottement interne avec l'augmentation du rapport (D $/ \mathrm{d}_{\max }$ ) ( $\mathrm{D}$ : diamètre de l'éprouvette et $\mathrm{d}_{\max }$ : diamètre du grain). [11] a trouvé des résultats similaires sur un mélange de sable et $20 \%$ de gravier $8 / 10 \mathrm{~mm}$ respectivement.

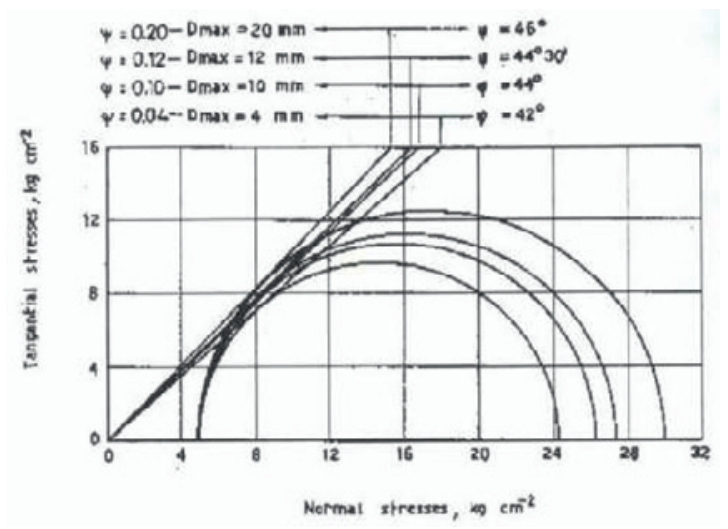

Fig. 3. Cercles de Mohr de rupture déduits d'essais triaxiaux pour différents valeurs de $\psi=\mathrm{d}_{\max } / \mathrm{D}$ (Fumagalli, 1969)

Sur la Figure 4, [10] a rassemblé les différentes résultats sur les valeurs de la cohésion et de l'angle de frottement interne issus de travaux antérieurs de la littérature en fonction de la dimension de la boîte L. Le diamètre maximal des grains varie de $5 \mathrm{~mm}$ jusqu'à $80 \mathrm{~mm}$ pour les différents matériaux étudiés. A cause de la nature assez différente des matériaux étudiés, on observe une forte dispersion des résultats, surtout en ce qui concerne la cohésion. En effet, la cohésion diminue avec l'augmentation du rapport de taille $\left(\mathrm{L} / \mathrm{d}_{\max }\right)$ et l'angle de frottement interne reste relativement constant pour toutes les tailles de boîte.
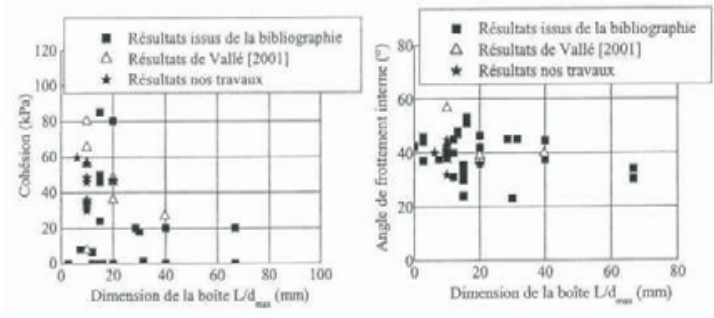

Fig. 4. Influence de la dimension de la boite sur la cohésion et l'angle de frottement interne (Afriani, 2003)

L'objectif est donc de réaliser des essais de cisaillement à l'aide de la boite de CASAGRANDE de section carrée $60 \times 60 \mathrm{~mm}$ au laboratoire sur des échantillons compactés à l'Optimum Proctor. Ces essais permettent de caractériser le comportement de deux sols grossiers (Tuf et Grave concassé) provenant des sites situés respectivement à $50 \mathrm{~km}$ (Mekkedra) et à $20 \mathrm{~km}$ (Sidi Ali_Benyoub) de Sidi Bel-Abbès, Ouest Algérien et d'étudier l'influence de la taille et de la nature des grains des sols testés sur la résistance au cisaillement. Pour réaliser ces essais de cisaillement direct, il est donc nécessaire tout d'abord de rappeler brièvement la connaissance donnée aux sols grossiers d'après différents auteurs. Un écrêtage par tamisage a été opéré de façon à réduire graduellement la dimension maximale du grain et opter pour les diamètres suivants : 20,10 et $5 \mathrm{~mm}$. Les résultats des essais attendus sont les caractéristiques de résistance au cisaillement à savoir l'angle de frottement et la cohésion du sol, paramètres de dimensionnement d'un tel ouvrage. Ces résultats seront présentés et comparés aux travaux de la littérature.

\section{Utilisation et connaissance d'un sol grossier}

Différents chercheurs ont défini différemment les sols grossiers ainsi que les limites de la taille des particules qui les composent en fonction des essais in situ ou en laboratoire [9].

[19] a été amené à étudier dans le cadre de travaux variés sur les barrages, la stabilité de versants, le comportement de sols grossiers. L'auteur a donc caractérisé le sol grossier par la taille des particules prédominantes dans le sol. Lambe et Whitman, cités par [19] considèrent comme un sol grossier, s'il contient plus de $50 \%$ de particules plus grandes que $75 \mu \mathrm{m}$. Selon le système de classification des sols grossiers par la British Standard Institution, 1981, (cité par [19]), les sols sont dits grossiers s'ils contiennent $65 \%$ de sables et de graviers dont la taille des grains est supérieure à $0,06 \mathrm{~mm}$. Par la suite, [20] définissent les sols grossiers comme ayant moins de $35 \%$ de matériau plus fin que $0,06 \mathrm{~mm}$. Ils confirment que ce sont des sols mal gradués. [21] pour des études de sol en laboratoire, ont travaillé avec des sols graveleux argileux destinés à des barrages en terre, des remblais et des revêtements en terre. Ils offrent une meilleure stabilité, un faible tassement et une grande résistance à l'érosion. Les particules de graviers étaient surtout composées de gneiss, de granite et de schiste, 
issus d'un dépôt de rivière. Les sols argileux de la matrice fine avaient un poids volumique qui variait de 26,6 à 27 $\mathrm{kN} / \mathrm{m}^{3}$. Pour l'étude des sols grossiers, les auteurs ont retenu la granulométrie des divers mélanges de sols graveleux comme indiqué sur la Figure 5.

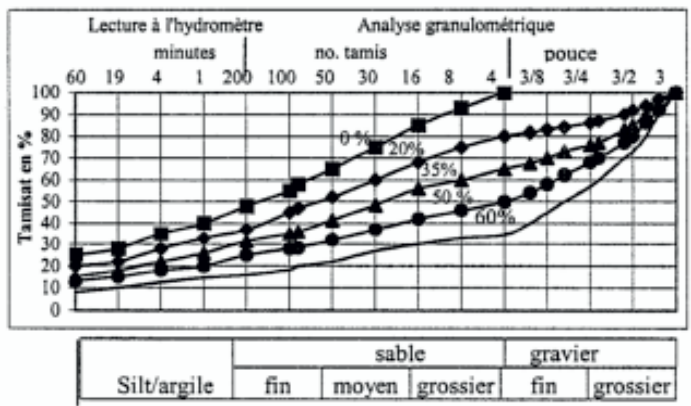

Fig. 5. Granulométries de sols graveleux argileux étudiés par Holtz et Willard, 1961

D'autres auteurs, [22] ont défini la taille des sols grossiers jusqu'à $50 \mathrm{~mm}$ de diamètre dans le cadre d'essais de résistance au cisaillement de matériaux grossiers en laboratoire. Ils ont effectué des essais in situ en utilisant des sols de dimension de particules allant jusqu'à 200 $\mathrm{mm}$. [23] a défini les sols grossiers qui contiennent plus de $50 \%$ d'éléments de taille supérieure à $2 \mathrm{~mm}$, la dimension limite supérieure des éléments n'étant pas fixée. Les sols grossiers les plus répandus sont issus de carrières ou bien, se trouvent dans la nature (éboulis, alluvions, ... ).

Par ailleurs, [7] a réalisés des essais qui ont permis de déterminer la dimension maximale des éléments du sol à étudier et a montré que cette dimension ne doit pas dépasser le rapport $\mathrm{L}_{\max } / 10\left(\mathrm{~L}_{\max }\right.$ plus grande dimension de la boite de cisaillement. [8] ont effectué des essais de cisaillement sur quatre classes de sols grossiers des Alpes selon le guide GTR, 2000 [24] sur la fraction 0/20 mm, on utilisant deux types de boitiers $(300 \times 300 \mathrm{~mm})$ et $(1000 \times 1000 \mathrm{~mm})$. La détermination des valeurs de la cohésion et de l'angle de frottement interne sont indiquées sur les courbes granulométriques qui sont réalisées sur la fraction $0 / 100 \mathrm{~mm}$ (Figure 6).

Les auteurs confirment la grande difficulté de la caractérisation du comportement mécanique des sols grossiers en particulier à l'aide de boitier de cisaillement direct de grande dimension.

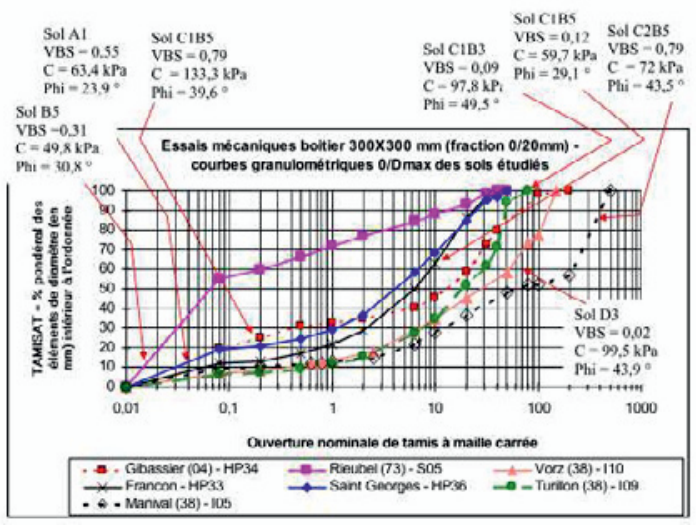

Fig. 6. Courbes granulométriques des sols étudiés de torrents $(0 / 100 \mathrm{~mm})$ par Tacnet et al., 2000

On peut dire que l'intervalle granulaire des sols grossiers a été discuté par beaucoup d'auteurs, surtout sur la limitation supérieure du diamètre des grains. A partir de nombreuses constatations, on peut situer les diamètres limites des sols grossiers. L'intervalle granulaire considéré va de 0,2 jusqu'à $50 \mathrm{~mm}$. D'une façon générale, ces seuils sont imposés par le respect de la représentativité de l'échantillonnage du sol et de l'essai réalisé avec les appareillages classiques de laboratoire [25].

\section{Matériaux et procédure expérimentale}

Les matériaux utilisés à savoir le Tuf et la Grave concassée, proviennent respectivement du gisement de Mekkedra et Sidi Ali-Benyoub situé à l'Ouest Algérien.

Ce choix s'explique par la proximité du site. Le prélèvement est effectué à la pelle dans une zone de dépôt stabilisé. Pour la réalisation des essais d'identification ainsi que les essais mécaniques de résistance, les deux matériaux ont été préparés à l'aide d'un écrêtage par tamisage de façon à réduire graduellement la dimension maximale du grain de manière à obtenir les fractions de l'étude à savoir :

- Tuf de Makkedra, on utilise les fractions $0 / 10 \mathrm{~mm}$; $0 / 5 \mathrm{~mm} ; 0 / 2 \mathrm{~mm}$.

- Grave concassée, on a choisi la fraction $0 / 5 \mathrm{~mm}$ afin de montrer l'influence de la nature du matériau sur la résistance au cisaillement.

Les résultats de l'analyse granulométriques des différentes fractions sont représentés dans la Figure 7. D'après la classification Guide des Terrassement Routier (GTR, 2000) [24], l'analyse granulométrique montre que les matériaux utilisés sont constitués essentiellement de graviers, de gros sable et très peu de sable fin. 


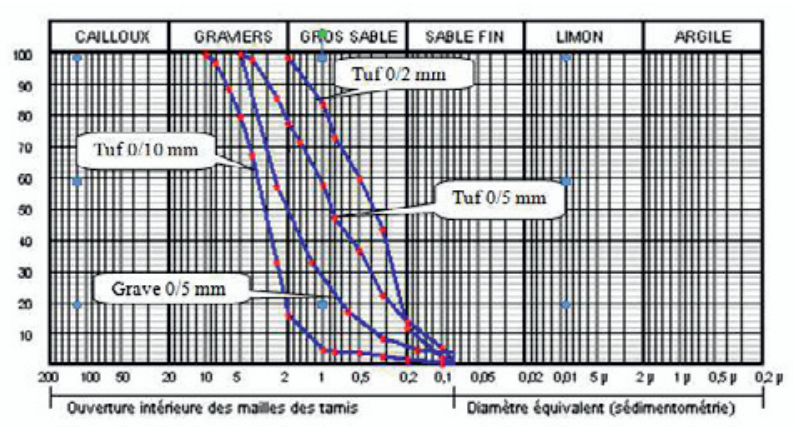

Fig. 7. Courbes granulométriques des sols étudiés

\subsection{Confections des éprouvettes}

Avant de procéder aux essais de cisaillement direct à la boite, nous avons réalisé des essais de compactage selon la norme (NF-P 94-093) [26] dans le moule Proctor (si $\mathrm{D} \leq 5 \mathrm{~mm}$ ) et dans le moule CBR (si $5<\mathrm{D} \leq 20 \mathrm{~mm}$ ) où $\mathrm{D}$ est le diamètre maximale des grains de chaque fraction choisie $(0 / 10 \mathrm{~mm} ; 0 / 5 \mathrm{~mm}$ et $0 / 2 \mathrm{~mm})$ afin de déterminer les Optimums Proctor (Tableau 1) qui vont servir à la confection des éprouvettes conçues pour la réalisation des essais de cisaillement selon la norme (NFP 94-071-1) [27]. Une fois le compactage réalisé, on découpe chaque éprouvette en galettes et seront imprégnées de paraffine (substance blanche faite d'un mélange d'hydrocarbures saturés solides caractérisée par leur inertie chimique, utilisée notamment dans la fabrication des bougies et de certains emballages) se qui leurs permettra de garder intact leurs caractéristiques optimales pendant plusieurs jours (Figure 8).

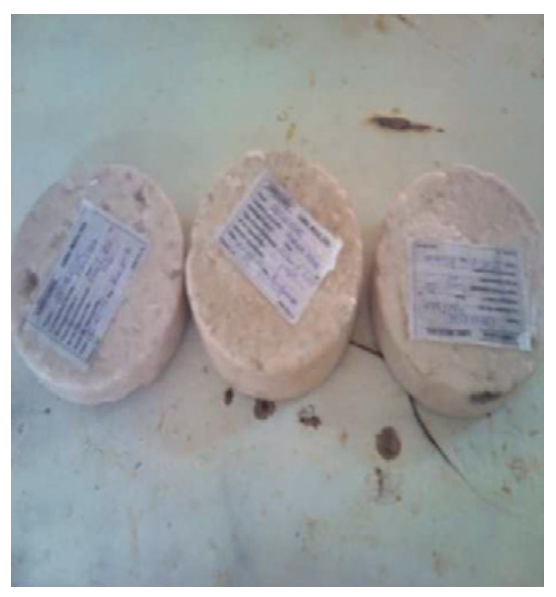

Fig. 8. Eprouvettes paraffinées sous forme de galettes

Tableau 1. Valeurs des caractéristiques à l'Optimum Proctor

\begin{tabular}{|c|c|c|c|c|}
\hline $\begin{array}{c}\text { Nature et classe } \\
\text { granulaire } \\
\text { du sol }\end{array}$ & $\begin{array}{c}\text { Tuf } \\
0 / 2 \\
\mathrm{~mm}\end{array}$ & $\begin{array}{c}\text { Tuf } \\
0 / 5 \\
\mathrm{~mm}\end{array}$ & $\begin{array}{c}\text { Tuf } \\
0 / 10 \\
\mathrm{~mm}\end{array}$ & $\begin{array}{c}\text { Grave } \\
\text { concassée } \\
0 / 5 \mathrm{~mm}\end{array}$ \\
\hline $\begin{array}{c}\text { Teneur en eau } \\
\text { optimum (\%) }\end{array}$ & 18.9 & 18.6 & 21.7 & 9.4 \\
\hline $\begin{array}{c}\gamma_{\mathrm{dmax}} \\
\left(\mathrm{g} / \mathrm{cm}^{3}\right)\end{array}$ & 1.54 & 1.58 & 1.65 & 2.18 \\
\hline
\end{tabular}

\subsection{Essai de cisaillement}

L'essai de cisaillement s'effectue sur une éprouvette de sol, de dimensions $60 \times 60 \times 20 \mathrm{~mm}$. Il faut tailler à l'aide de la trousse coupante les éprouvettes de telle sorte que les dimensions de la section droite de l'éprouvette soient ajustées au mieux à celle de la boite. L'éprouvette est calée dans la boite de telle sorte que le plan de glissement la partage en deux parties sensiblement égales. Après la mise sous contrainte vertical de l'éprouvette, la demiboîte inférieure est entraînée horizontalement à une vitesse de cisaillement constante de $0,5 \mathrm{~mm} / \mathrm{min}$. Lors de essai, on mesure la force totale $\mathrm{F}$ de cisaillement, le déplacement horizontal et le déplacement vertical (Figure 9). L'essai nécessite le cisaillement d'au moins trois éprouvettes de mêmes dimensions, préparées dans les mêmes conditions et soumises à des contraintes verticales de $100 \mathrm{kPa}, 200 \mathrm{kPa}$ et $300 \mathrm{kPa}$. L'essai choisi est un essai non consolidé non drainé (UU).

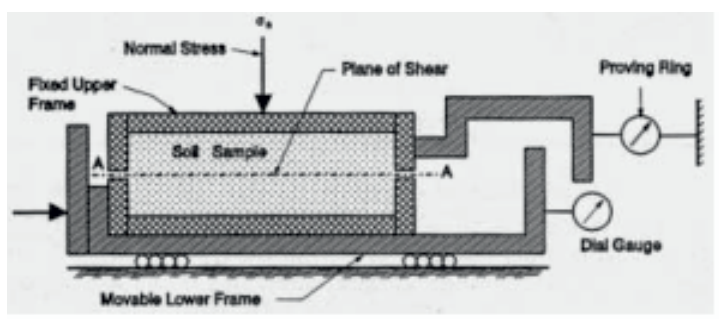

Fig. 9. Principe de l'essai de cisaillement rectiligne

La détermination des paramètres mécaniques des différentes éprouvettes de cisaillement s'obtient en traçant les droites de coulomb (courbes intrinsèques) dans un repère orthonormé qui représente, en abscisse les contraintes verticales $(\sigma)$ et en ordonné les contraintes de cisaillements maximales $\left(\tau_{\max }\right)$. Les pentes de ces courbes représentent les tangentes des angles de frottement interne $\boldsymbol{\varphi}$ d'une part, et d'autre part les cohésions c s'obtiennent par l'intersection de ces droites avec l'axe des ordonnés.

\section{Résultats et discussions}

Les résultats des essais de cisaillement montrent que la taille des grains et la nature du sol ont une influence sur le comportement mécanique de résistance au cisaillement. Il a été constaté pour les deux matériaux compactés initialement à l'OPN qu'il n' ya pas de pic de résistance sur les courbes contraintes-déplacements, rarement un palier (Figures 10, 11, 12 et 13). Ceci confirme d'autres résultats obtenus de la littérature sur les sols grossiers [28]; [29]; [6]. L'interprétation des résultats et la représentation du critère de rupture par les droites de Mohr Coulomb peuvent être discutées. 


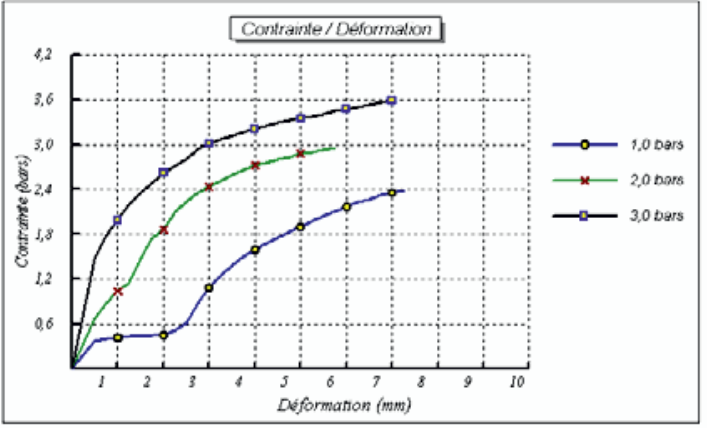

Fig. 10. Courbes de résistance au cisaillement du tuf $0 / 2 \mathrm{~mm}$

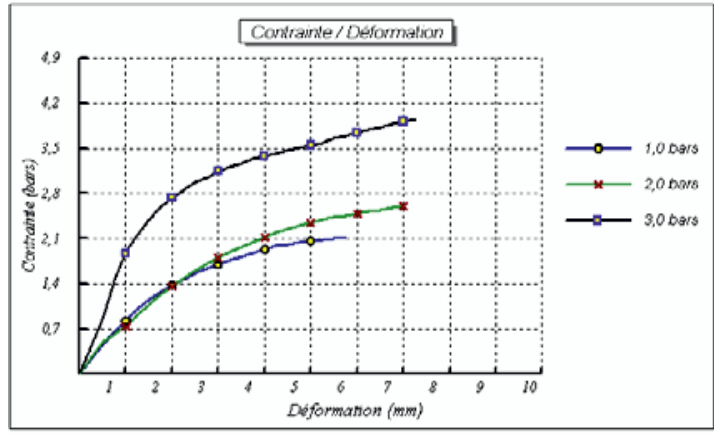

Fig. 11. Courbes de résistance au cisaillement du tuf $0 / 5 \mathrm{~mm}$

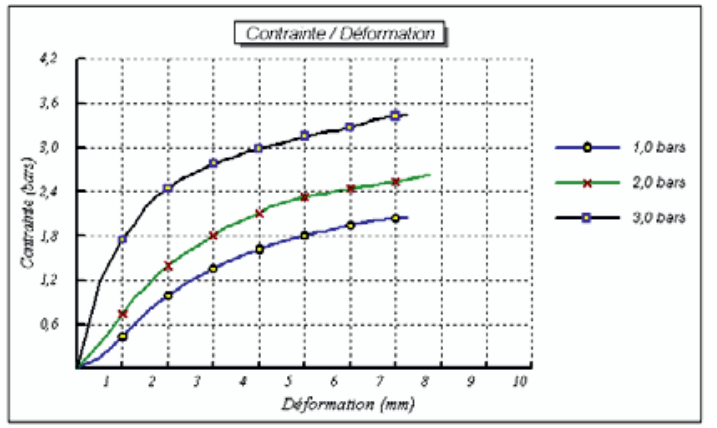

Fig. 12. Courbes de résistance au cisaillement du tuf $0 / 10 \mathrm{~mm}$

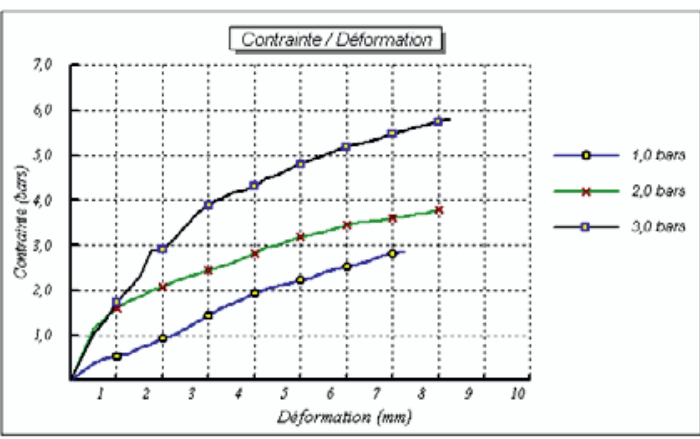

Fig. 13. Courbes de résistance au cisaillement de la Grave concassée $(0 / 5 \mathrm{~mm})$

\subsection{Tuf de Mekkedra}

On a étudié l'influence de la technique d'écrêtage ou enlèvement de la fraction grossières sur les paramètres mécaniques comme la cohésion et l'angle de frottement interne. Les classes granulaires mises en œuvre sont $0 / 2 \mathrm{~mm}, 0 / 5 \mathrm{~mm}$ et $0 / 10 \mathrm{~mm}$. La comparaison des courbes de cisaillement sur les matériaux écrêtés qui représentent la cohésion et l'angle de frottement interne en fonction de $\mathrm{d}_{\max }$ sont montrés dans les Figures 14 a et $\mathrm{b}$. Une augmentation de la résistance maximale est observée avec l'augmentation de la taille du seuil d'écrêtage (Figure 14 a). On constate que l'angle de frottement interne $\varphi$ augmente en fonction de l'augmentation de la taille des grains. En revanche, la cohésion c diminue lorsque le diamètre de la particule $\left(\mathrm{d}_{\max }\right)$ augmente (Figure $14 \mathrm{~b}$ ). Ces résultats sont cohérents avec la littérature [1]; [9]; [10]; [11] et [30]. D'autres résultats [13]; [31] montrent une faible influence sur les caractéristiques à la rupture du matériau grossier. En revanche, [30] montrent que l'engrènement des particules grossières génère une cohésion apparente et on peut dire que la cohésion croît avec l'augmentation du paramètre $\mathrm{d}_{\max }$.

Par ailleurs, l'extrapolation de la courbe (cohésiondiamètre maximal) conduit à un angle de frottement intergranulaire $\boldsymbol{\varphi}_{\mathbf{i}}$ de l'ordre de $28,6^{\circ}$ correspondant à un diamètre $\mathrm{d}_{\max }=0$. Ce résultat tend vers un matériau "idéal" tel que $d_{\max }=0$ et la cohésion c est presque nulle, de l'ordre de $1,13 \mathrm{kPa}$. Cette interprétation permet d'écrire la résistance au cisaillement du matériau sous la forme :

$$
\begin{gathered}
\tau_{f}=c\left(d_{\max }\right)-c_{0}+\sigma_{n} \tan \left(\phi\left(d_{\max }\right)\right) \\
\text { où : } c\left(d_{\max }\right)=c_{0}+\phi_{l}+\alpha_{c} d_{\max } \\
\tan \phi\left(d_{\max }\right)=\tan \phi_{l}+\alpha \phi d_{\max }
\end{gathered}
$$

La résistance au cisaillement normalisée par l'état de contrainte verticale s'écrit sous la forme :

$\tau_{f} / \sigma_{n}=\tan \phi+\left(c-c_{0}\right) / \sigma_{n}$

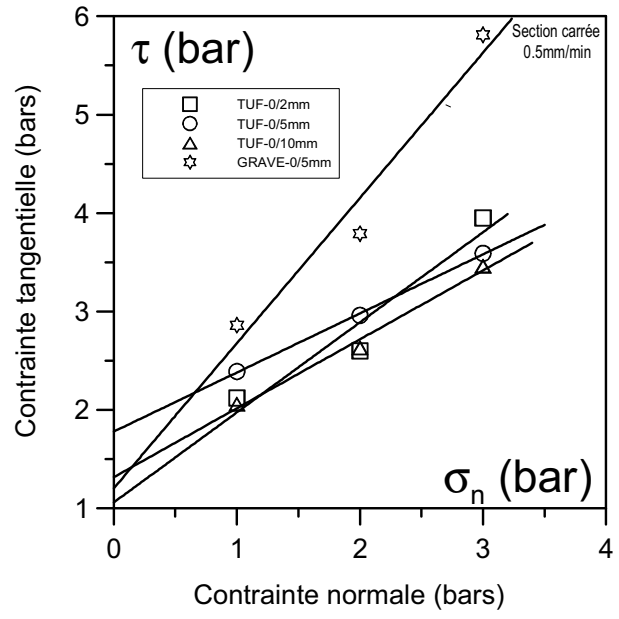

Fig. 14.a Détermination de l'angle de frottement et de la cohésion des deux matériaux testés 


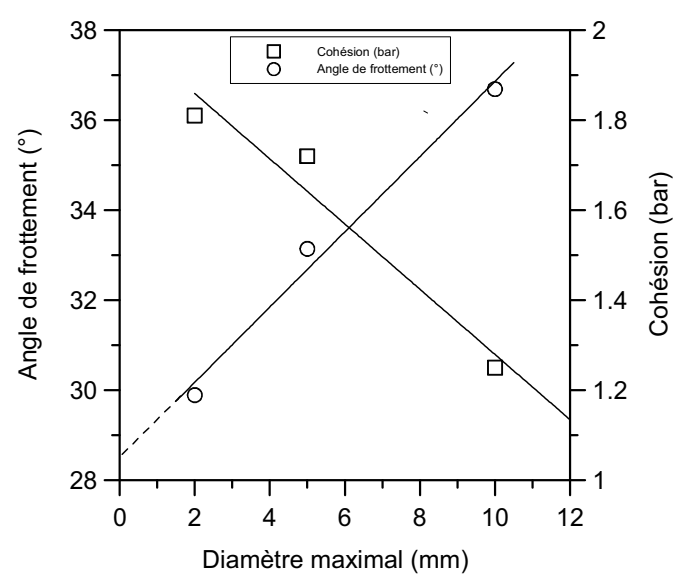

Fig. 14.b Effet de la taille du grain sur l'angle de frottement et la cohésion du tuf de Mekkedra

\subsection{Grave concassée}

On observe une résistance au cisaillement plus élevé que le tuf (Figure 14 a). ce qui justifie l'influence de la nature du matériau. Cela signifie une augmentation de l'angle de frottement interne de $54,78^{\circ}$ et une cohésion identique à celle trouvée sur le matériau tuf de classe $0 / 10 \mathrm{~mm}$ qui de l'ordre de $1.21 \mathrm{kPa}$, vu que la grave concassé est un sol constitué essentiellement de gros éléments et très peu d'éléments fins. Ce terme de cohésion correspond plus à un enchevêtrement des grains entre eux, plutôt qu'à une adhésion des particules fines.

Il est donc très important de pouvoir caractériser les sols grossiers au laboratoire à l'aide de dispositifs de taille classique avec un rapport $\left(\mathrm{L} / \mathrm{d}_{\max }\right) \geq 10$. Cela nécessite de tester un volume de sol suffisamment grand pour qu'il soit représentatif du sol in situ, tout en étudiant l'influence de la fraction volumique des inclusions. Ce volume est définie par la méthode d'écrêtement.

Notre étude nous montre que la taille des particules des sols grossiers que nous avons utilisés a une influence marquée sur le comportement mécanique de résistance au cisaillement. Des points très importants ont été observés concernant la taille des gros éléments et nature du sol :

- $\quad$ On remarque que pour le tuf de Mekkedra, l'angle de frottement $\varphi$ augmente en fonction de classe granulaire $\mathrm{L} / \mathrm{d}_{\max } \mathrm{du}$ sol c'est-à-dire plus la classe granulaire (augmentation des gros éléments) du sol augmente, plus l'angle de frottement du matériau augmente.

- Cependant, on remarque que la cohésion c diminue en fonction de l'augmentation de la classe granulaire $\mathrm{L} / \mathrm{d}_{\max }$.

- $\quad$ On observe aussi pour la grave concassée que la résistance au cisaillement est plus élevé, comparativement au tuf. Cela peut s'expliquer par la nature du matériau car la grave concassé est un sol constitué d'essentiellement de gros éléments et peu d'éléments fins, ce qui signifie une augmentation de l'angle de frottement et une diminution la cohésion du matériau.

\section{Conclusions}

Pour étudier le comportement mécanique des sols grossiers à l'aide de dispositifs de laboratoire de taille classique, on doit, le plus souvent, écrêter ou substituer une partie du sol.

Afin de caractériser les sols grossiers, il est très important d'étudier l'influence des paramètres de base sur leur comportement mécanique à matrice en terme de résistance au cisaillement, comme l'influence de la taille des grains et la nature du matériau.

Nous avons présenté dans cet article les résultats d'un programme d'essais réalisé sur la boîte classique de cisaillement direct, pour étudier le comportement mécanique des sols grossiers en terme de résistance au cisaillement. Ces sols grossiers sont compactés à une teneur en eau optimum déterminée par des essais de Proctor normal. Les résultats obtenus sur ces sols grossiers montrent l'effet apporté par la taille des gros éléments sur leur résistance au cisaillement. Deux points importants ont été observés :

$\checkmark$ L'angle de frottement augmente de manière significative avec l'augmentation de la taille des gros éléments. Cette augmentation est bien marqué lorsqu'on utilise un matériau plus dur avec le même diamètre maximal $\left(\mathrm{d}_{\max }\right)$. Ces résultats sont cohérents avec ceux trouvés par [10]; [11]; [30] et [31].

$\checkmark$ La cohésion diminue en fonction de l'augmentation de la taille des gros éléments. Contrairement à certains chercheurs comme [9] et [10] qui ont trouvé que la cohésion augmente avec l'augmentation des diamètres. La diminution de la cohésion peut être due par la diminution de la qualité de sable fin contenu dans l'échantillon au fur et à mesure de l'augmentation des gros éléments.

\section{Références}

1. Fumugalli,E, 1969. Tests on cohesionless materials for rockfill dams. Journal of the soil mechanics and foundations division. ASCE. 95, n℠1, pp. 313-330

2. Patwardhan A S. Shivaji Rao J. Gaidhane R B, 1970. Interlocking effects and shearing resistance of boulders and large size particles in A matrix of fines on the basis of large scale direct shear tests. The second south-east Asian Conference on Soils Engineering. Singapure, pp. 265-73.

3. Ortigosa P. Musante H. Kort I, 1981. Mechanical proprieties of the gravel of Santiago. Proceeding of the tenth International Conference on Soils Mechanics and Foundation Engineering. Stockholm, Vol. II, p. 545-548.

4. Ramamurthy T et Gupta K K, 1980. Prediction of the behavior of rockfillmaterials. Third Australia New Zealand Conference on geomechanics. Wellington, Vol. 1, pp 1-13.

5. Fry J. Mamba M. et Flavigny E, 1989. Classification et propriétés des enrochements: Le cas d'un grès, Rio de Janeiro, Vol. 1, pp. 713-714. 
6. Shirdam R, 1998. Comportement mécanique des matériaux superficiels des versants naturels. Thèse de doctorat, Institut National des Sciences Appliquées de Lyon, 216 p.

7. Guichard, J. C, 1994 Etude bibliographique et expérimentale sur les caractéristiques mécaniques des matériaux grossiers. Mémoire de DEA, INSA Lyon, Cemagref Aix-en Provence, France.

8. Tacnet, J. M, Gotteland, Ph, Bernard A, Mathieu G et Deymier C., 2000. Mesure des caractéristiques géotechniques des sols grossiers. Application aux sols de Torrent. Internationales Symposion. Interpraevent $2000 \quad-\quad$ Villach/Österreich, Tagungspublikayion, Band 3, Seite 307-319.

9. Valle N, 2001. Propriétés mécaniques d'un sol grossier d'une terrasse alluvionnaire de la Seine. Thèse de doctorat, Université de Caen, 304 p.

10. Afriani L, 2003. Essais de cisaillement direct des sols grossiers : Incidences des procédures d'essai et effets d'échelle. Thèse de doctorat, Université de Caen. $184 \mathrm{p}$.

11. Pedro L, 2004. Etude du comportement mécanique de sols hétérogènes modèles à son application au cas des sols naturels. Thèse de doctorat, Ecole Nationale des Ponts et Chaussées, 294 p.

12. Cruz P. T. et Nieble C.N, 1971. Engineering properties of residual soils and granular materials originated from Basalt - Capivari Dam-Bresil. Proceeding of the Fifth Regional Conference for Africa on Soil Mechanics and Foundation Engineering, Luanda, Vol. 1, pp. 143-149.

13. Donagh R. T. et Torrey V. H, 1979. Scalping and replacement effects on strength parameters of earthrock mixtures. In BGS, editor, Design Parameters in geotechnical engineering, London, Vol. 2, pp. 29-34.

14. Zhang Q.Y. et Si H.Y, 1982. Shear strength and stress strain properties of coarse-grain soil. Proceedings of the Seventh South East Asian Geotechnical Conference, Hong Kong, Vol. 1, pp. 931-945.

15. Torrey, V.H. et Donagh, R.T, 1985. Strength parameters of earth-rock mixtures. Proceedings of the eleventh International Conference on Soil Mechanics and Foundation Engineering. San Francisco, Vol. 2, pp. 1073-1076.

16. Sharma V M. Venkatachalam K et Roy A, 1994 Strength and deformation Characteristics of rockfill Materials in Urac. Proceedings, Thirteenth International Conference on soils Mechanics and Foundation Engineering. New Delhi, Vol. 3, pp 959962.

17. Yagiz S, 2001. Brief note on the influence of shape and percentage of gravel on the shear strength of sand and gravel mixtures. Bulletin of Engineering Geology and the Environment, Vol. 60, 4, pp. 321323.

18. Tacnet, J. M et Gotteland, Ph, 1997. Amélioration de la connaissance des caractéristiques géotechniques des sols grossiers. Rapport $1^{\text {ère }}$ phase (1996-1997). Cemagref, Division Erosion Torrentielle Neige avalanches.
19. Charles, 1A, (1989), General report / discussion session 8: geotechnical properties of coarse grained soil, Proceedings of the Twe1fth International Conference on Soil Mechanics and Foundation Engineering, Rio de Janeiro, volume 4, pp. 2495 2519.

20. Craig, R.F. et Susilo, B.S, 1986. Mekanika tanah, Erlanga éditeur, $374 \mathrm{p}$.

21. Holtz W. G. et Willard E, 1961. Triaxial shear characteristics of clayed gravel soils. Comptes rendus, 5ème Congrès International de Mécanique des Sols et Travaux de Fondation, Paris, pp. 143149

22. Nichiporovitch, AA et Rasskazov, L.N., (1967), Shear strength of coarse fragmentaI materials, Proceedings of the Geotechnical Conference, Oslo, volume 1, pp. 225-229.

23. Perrot, A, (1968), Etude de la résistance au cisaillement des matériaux grossiers, Rapport interne du laboratoire des Ponts et Chaussées, Nancy, $40 \mathrm{p}$.

24. G.T.R, 2000. Guide Technique pour la réalisation des remblais et des couches de forme, fascicule I, principes généraux, 2000. 166p.

25. Afriani. L, Levacher. D, Khay. $M$ et Lozach. D, 2002. Résistance au cisaillement d'une grave alluvionnaire - essai à la boite : constats et effet d'échelle. VII ème Journée Nationale Génie Civil Génie Côtier, Anglet, France, 15-17 Mai.

26. NF $P$ 94-093. Détermination des références de compactage d'un matériau. Essai Proctor normal. Essai Proctor modifié. 18 p.

27. NF P 94-071-1. Essai de cisaillement rectiligne à la boîte. Partie 1: cisaillement direct.16 p.

28. Bourdeau, Y, 1997. Le comportement des alluvions du Rhône dans une grande boite de cisaillement direct. Revue française de géotechnique, $\mathrm{n}^{\circ} 79$; $\mathrm{pp}$ 45-57.

29. Gotteland, P., Tacnet, J. M, et Aboura, A. 2000. Caractérisation des sols grossiers, application aux sols de torrents. revue Française de génie civil, vol. 4(2-3), pp.25-338.

30. Reiffsteck, P., Nguyen Pham, P.-T, Arbaut, J., 2007. Influence de la répartition granulaire sur le comportement mécanique d'un sol. Bulletin des laboratoire des ponts et chaussées. pp 83-104.

31. Holtz, W. G., Gibbs, J., 1956. Triaxial mechanics tests on previous gravelly soils. Journal of the soil mechanics and foundation division, ASCE, vol. 82, paper 867 , pp. 1-22. 